\title{
Composição e diversidade florístico-estrutural de um hectare de floresta densa de terra firme na Amazônia Central, Amazonas, Brasil'
}

\author{
Arlem Nascimento de OLIVEIRA², Ieda Leão do AMARAL ${ }^{3}$, Michele Braule Pinto RAMOS4, \\ Antônio Donato NOBRE5, Luciana Bovino COUTO ${ }^{3}$, Rosana Martiniano SAHDO ${ }^{3}$
}

\begin{abstract}
RESUMO
Foram inventariadas todas as árvores, lianas e palmeiras com DAP $\geq 10 \mathrm{~cm}$ de um hectare (dois transectos paralelos de 500 x $10 \mathrm{~m})$ de floresta densa de terra firme sobre platô de Latossolo, $90 \mathrm{~km}$ a nordeste de Manaus $\left(02^{\circ} 35^{\prime} 45^{\prime \prime} \mathrm{S}\right.$ e $60^{\circ} 12^{\prime} 40^{\prime \prime}$ W). A fitofisionomia local é exuberante e homogênea, com grande número de árvores altas e finas. Foram encontrados 670 indivíduos distribuídos em 48 famílias, 133 gêneros e 245 espécies. Do total amostrado, 70\% ou 467 indivíduos apresentaram $\mathrm{DAP} \leq 22,1 \mathrm{~cm}$. Abarema mataybifolia (Sandw.) Barneby \& Grimes, Leonia glycycarpa Ruiz \& Pav., Swartzia reticulata Ducke e Aspidosperma oblongum A. DC., foram as únicas espécies a apresentarem valores superiores a $90 \mathrm{~cm}$ de DAP. Fabaceae, Sapotaceae e Lecythidaceae constituíram as três famílias com maior riqueza de espécies e maiores índices de valor de importância aos níveis de família e espécie. Os índices de diversidade $\left(\mathrm{H}^{\prime}=5,1\right)$ e de equitabilidade $\left(\mathrm{E}^{\prime}=0,92\right)$, ambos de Shannon-Wiener, indicam que a floresta é bem diversificada, com uma abundância relativamente uniforme das espécies. Nesse ambiente florestal, as espécies não tem distribuição espacial uniforme, porém, quanto menor a distância geográfica entre as subparcelas, maior sua similaridade florística (teste de Mantel, $\mathrm{p}<0,001$ ).
\end{abstract}

PALAVRAS-CHAVE: estrutura fitossociológica, diversidade, similaridade, terra firme, Amazônia Central

\section{Composition and floristic-structural diversity of a hectare of terra firme dense forest in Central Amazonia, Amazonas, Brazil}

\begin{abstract}
To investigate the composition and floristic diversity of one hectare of a dense forest on a terra firme oxisol plateau, $90 \mathrm{~km}$

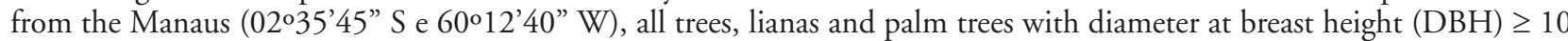
$\mathrm{cm}$ were inventoried along two parallel transects of $500 \times 10 \mathrm{~m}$. The landscape is vegetationally exuberant and homogeneous, with a large quantity of tall slender trees. A total of 670 individuals in 48 families, 133 genera and 245 species were registered in this floristic inventory. 467 of the plants sampled exhibited DBH $\leq 22.1 \mathrm{~cm}$, representing $70 \%$ of the total. Abarema mataybifolia (Sandw.) Barneby \& Grimes, Leonia glycycarpa Ruiz \& Pav., Swartzia reticulata Ducke and Aspidosperma oblongum A. DC. were the species with DBH $>90 \mathrm{~cm}$. Families with greatest species richness and importance value were Fabaceae, Sapotaceae and Lecythidaceae. The Shannon-Wiener diversity $\left(\mathrm{H}^{\prime}=5.1\right)$ and evenness $\left(\mathrm{E}^{\prime}=0.92\right)$ indices suggest that the forest environment is very diversified, but with a relative uniformity of species. However, a uniform spatial distribution of the species in this forest environment was not observed. According to Mantel's test $(\mathrm{p}<0.001)$, the highest floristic similarity is a function of geographic proximity among suplots.
\end{abstract}

KEY WORDS: phytosociology, diversity, similarity, terra firme, Central Amazonia

\footnotetext{
1 Trabalho executado com auxilio financeiro do CNPq, PPD-G7/LBA.

${ }^{2}$ Universidade Federal do Amazonas/DCFDA, Manaus, Amazonas, Brasil. arlem@inpa.gov.br; arllem@yahoo.com.br.

${ }^{3}$ Instituto Nacional de Pesquisas da Amazônia/CPBO.

${ }^{4}$ Instituto Nacional de Pesquisas da Amazônia/CPST. Av. André Araújo, 2936, Petrópolis, CEP 69060-001, C. Postal 478, Manaus, AM, Brasil

${ }^{5}$ Instituto Nacional de Pesquisas Espaciais-INPE.
} 


\section{INTRODUÇÃO}

A fragilidade dos ambientes florestais da Amazônia indica a necessidade prioritária de se conhecer a composição e distribuição das espécies vegetais nesses ecossistemas, visando com isso planificar de forma eficiente o manejo e a conservação da flora nativa regional. Nesse sentido, os estudos florístico e fitossociológico são imprescindíveis, pois a partir de informaçôes quali-quantitativas, pode-se conhecer, entre outros, a função das diferentes espécies de plantas na comunidade, bem como os habitats preferenciais de cada uma delas.

Vários trabalhos concluídos na Amazônia Ocidental e Central têm revelado que as florestas de terra firme possuem alta diversidade de espécies com árvores de DAP maiores ou iguais a $10 \mathrm{~cm}$, e grande percentual de espécies com apenas um indivíduo por hectare, além de baixa similaridade florística entre parcelas próximas (Campbell et al., 1986; Gentry, 1988; Campbell, 1994; Valência et al., 1994; Amaral, 1996; Ferreira \& Prance, 1998; Lima Filho, 2001).

Fatores climáticos e edáficos como climas mais chuvosos e menos sazonais, e solos relativamente mais férteis em nutrientes, têm sido citados como possíveis responsáveis por uma suposta maior diversidade de plantas na Amazônia Ocidental (Gentry, 1988; Clinebell et al., 1995; ter Steege et al., 2000). Outros autores (Phillips et al., 1994) relacionam a maior riqueza de espécies à dinâmica natural de mortalidade de árvores, onde florestas com altas taxas de mortalidade e recrutamento seriam mais diversificadas.

Três inventários de um hectare, com DAP mínimo de 10 cm, realizados na Amazônia Central (Oliveira \& Mori, 1999a) revelaram uma riqueza de espécies muito próxima àquela registrada nos inventários da Amazônia Ocidental, porém, em condições de precipitação marcadamente sazonal e em solos de baixa fertilidade. Esses resultados encontrados em parcelas da Amazônia Central contradizem as relaçôes estabelecidas entre diversidade de árvores, precipitação, sazonalidade e dinâmica (Oliveira, 2000). Para Oliveira \& Daly (1999b), essa alta diversidade pode estar associada a uma confluência de regiôes fitogeográficas distintas, congregando espécies provenientes de diferentes regiôes.

A dissimilaridade entre comunidades vegetais na Amazônia Ocidental está associada, em parte, com a topografia e as características edáficas (Guillaumet, 1987; Gentry, 1988; Tuomisto et al., 1995). Outros autores observaram que, mesmo entre parcelas muito próximas, pode ocorrer baixa similaridade em florestas de terra firme (Campbell et al., 1986; Campbell, 1994; Ferreira \& Prance, 1998); os diferentes índices empregados nesses estudos (Jaccard, Sorensen e Porcentagem) variaram de $10-36 \%$ de similaridade florística, sugerindo que não deve existir uma amostragem pequena representativa para nenhuma floresta amazônica em particular
(Campbell, 1994). Em outras palavras, essa baixa sobreposição de espécies pode ser fruto de uma amostragem incompleta da comunidade, em parcelas de um hectare.

Nota-se escassez de estudos empregando índices de similaridade entre parcelas em florestas terra firme da Amazônia Central (Ferreira \& Prance, 1998; Oliveira \& Amaral, 2004). O presente trabalho, além de abordar aspectos florísticos e estruturais da vegetação, avaliará a influência da distância geográfica sobre as similaridades florísticas entre as subparcelas, em uma floresta de platô da Amazônia Central.

\section{MATERIAL E MÉTODOS}

\section{ÁREA DE ESTUDO}

A Estação Experimental ZF-2 do Instituto Nacional de Pesquisas da Amazônia, INPA, com área aproximada de 600 $\mathrm{km}^{2}$ localiza-se à noroeste da cidade de Manaus, AM, tendo como limites a Rodovia BR-174 (Manaus - Boa Vista) e a bacia do Rio Cuieiras (Figura 1); a altitude do ambiente florestal estudado é de aproximadamente 40 a $100 \mathrm{~m}$.

O clima dessa região é "Am" na classificação de Köppen (RADAM, 1978), com precipitação anual entre 1.355 a $2.839 \mathrm{~mm}$. A temperatura média varia de 25,6 a $27,6^{\circ} \mathrm{C}$, com umidade relativa do ar variando de 84 a $90 \%$ ao longo do ano; os meses mais chuvosos são de dezembro a maio, e os mais secos de agosto a novembro.

Os solos são classificados como Latossolo Amarelo Álico, de textura argilosa, caracterizado pela presença de um horizonte $\mathrm{B}$, poroso, fortemente micro-agregado. O material original desses solos é composto pelos sedimentos terciários do grupo Barreiras, essencialmente constituídos de minerais, tais como, caulinita, quartzo, óxidos e hidróxidos de ferro e alumínio (Ranzani, 1980; Chauvel, 1982). Apresentam

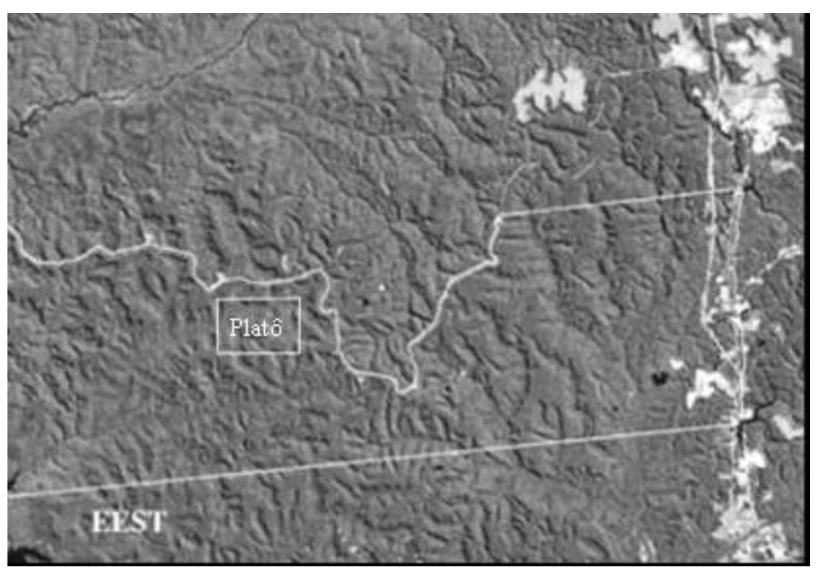

Figura 1 - Imagem de Satélite LandSat 7 da Estação ZF-2, INPA, Banda 3, 4 e 5 (RGB), 13/07/1999 (Fonte: INPE). Coordenadas: 2035'45" S e 60²'40" W (Fonte: GISLAB). 
ainda um perfil profundo, ácido e de baixa fertilidade natural (Ranzani, 1980).

\section{COLETA E ANÁLISE DOS DADOS}

Para o inventário florístico, amostraram-se dois transectos de $10 \times 500 \mathrm{~m}$, paralelos e distantes $200 \mathrm{~m}$ entre si. Cada transecto foi dividido em dez parcelas primárias de $10 \times 50$ $\mathrm{m}$, dentro das quais mensuraram-se os indivíduos arbóreos, palmeiras e lianas com diâmetro à altura do peito (DAP) $\geq 10$ $\mathrm{cm}$. Durante as mensurações, realizaram-se ainda observaçôes de toda vegetação ao longo das parcelas avaliadas, quanto ao porte e diâmetro dos indivíduos e grau de penetração de luz, visando obter informaçôes fisionômicas do ambiente florestal estudado. A altura do fuste (distância da superfície do solo até a primeira bifurcação ou até a inserção dos primeiros galhos) das árvores foi determinada com auxílio de uma trena graduada até $50 \mathrm{~m}$. A altura total (altura do fuste + altura da copa) também foi obtida por comparação com as árvores mais altas de cada parcela que foram medidas por meio da escalada no fuste.

As amostras botânicas, férteis (flores e frutos) ou não, foram submetidas à secagem em estufa $\left(70^{\circ} \mathrm{C}\right)$ por 48 horas. Em seguida, essas amostras foram identificadas por meio da morfologia comparada com as exsicatas disponíveis no herbário do INPA e de consultas à literatura especializada em espécies florestais nativas da Amazônia Central (Ribeiro et al., 1999). As amostras não identificadas com nomes científicos receberam códigos de morfotipo. Após esse processo, o material fértil foi incorporado ao acervo do referido herbário. Os nomes botânicos apresentados nesse estudo foram conferidos com a página da WEB do Missouri Botanical Garden (http://mobot. mobot.org/W3T/Search/vas.html).

\section{AVALIAÇÕES ESTRUTURAIS}

Para avaliar os parâmetros fitossociológicos (MüellerDombois \& Ellenberg, 1974), estimaram-se a área basal (G) da área amostrada, densidades absoluta (DA) e relativa (DR), freqüências absoluta (FA) e relativa (FR), dominâncias absoluta (DoA) e relativa (DoR) e, por fim, o índice de valor de importância (IVI). A estimativa da importância ecológica das famílias no ecossistema estudado foi feita mediante o índice de valor de importância familiar (IVIF), calculado pela soma da diversidade ( $\mathrm{n}^{\circ}$ de espécies da família / $\mathrm{n}^{\circ}$ total de espécies), densidade e dominância relativas (Mori \& Boom, 1983). Para tais análises, foi utilizado o programa Microsoft EXCEL for Windows.

\section{DIVERSIDADE FLORÍSTICA}

As diversidades florísticas foram avaliadas por meio dos índices de riqueza de espécie e de Shannon-Wiener (Magurran, 1988). O primeiro índice (S/A) foi obtido da razão entre o número de espécies acumuladas e o tamanho da área amostrada $\left(\mathrm{m}^{2}\right)$, enquanto o segundo, pela fórmula: $\mathrm{H}^{\prime}=-\Sigma$ $\left(\mathrm{p}_{\mathrm{i}}\right)\left(\log _{\mathrm{e}} \mathrm{p}_{\mathrm{i}}\right)$, onde $\mathrm{p}_{\mathrm{i}}=(\mathrm{ni} / \mathrm{N})$ é a probabilidade de que um indivíduo amostrado aleatoriamente pertença a espécie $\mathrm{i} ; \mathrm{n}_{\mathrm{i}}=$ $\mathrm{n}^{\circ}$ total de indivíduos da espécie i; $\mathrm{N}=\mathrm{n}^{\circ}$ total de indivíduos amostrados na área; a equitabilidade (uniformidade) foi obtida segundo a fórmula $E^{\prime}=H^{\prime} / \log _{\mathrm{e}} S$ (Magurran, 1988), onde $\mathrm{S}=\mathrm{n}^{\mathrm{o}}$ de espécies.

Calculou-se, ainda, o $\alpha$ de Fisher (Krebs, 1989) desse, e de outros trabalhos, desenvolvidos em solos de terra firme que fornecessem o número de indivíduos amostrados e de espécies identificadas com diâmetro mínimo de inclusão $\geq 10 \mathrm{~cm}$. A fórmula utilizada foi: $S / N=[(1-x) / x]\left[-\log _{e}(1-x)\right]$, onde $S$ e $\mathrm{N}$ = Número total de espécies e indivíduos, respectivamente, na amostra; $\mathrm{x}=$ Parâmetro da série logarítmica; $\alpha \mathrm{x}, \alpha \mathrm{x}^{2} / 2$, $\alpha x^{3} / 3, \alpha x^{4} / 4, \ldots$, onde $\alpha x=$ Número de espécies no total representada por um indivíduo; $\alpha \mathrm{x}^{2} / 2=$ Número de espécies representada por dois indivíduos, e assim sucessivamente.

\section{SIMILARIDADE FLORÍSTICA E ANÁLISE DE AGRUPAMENTO}

As similaridades florísticas entre as 20 parcelas foram estimadas a partir dos índices quantitativo de Porcentagem e qualitativo de Sorensen (Müeller-Dombois \& Ellenberg, 1974), com esse último sendo calculado mediante a fórmula $\mathrm{Cs}=[2 \mathrm{c} /(\mathrm{a}+\mathrm{b})] \mathrm{x} 100$, onde $\mathrm{c}=\mathrm{n}^{\circ}$ de espécies comuns às duas parcelas; $\mathrm{a}$ e $\mathrm{b}=\mathrm{n}^{\circ}$ de espécies presentes somente nas parcelas 1 e 2, respectivamente. As análises de agrupamento entre parcelas foram feitas a partir do método UPGMA (Krebs, 1989), considerando inicialmente todas as espécies presentes na amostragem e em seguida, excluindo as espécies com apenas um indivíduo no hectare estudado. Nessas análises, o programa MVSP versão 3.1 for Windows (www.kovcomp.co.uk/mvsp) foi usado para gerar os dendogramas.

Foi utilizado, ainda, o teste de Mantel (1967) para avaliar a influência da distância geográfica entre parcelas sobre a similaridade florística, comparando duas matrizes independentes: similaridade florística e as distâncias (m) entre as 20 parcelas. A distância mínima e máxima entre as parcelas foi de 50 e $650 \mathrm{~m}$, respectivamente.

\section{RESULTADOS E DISCUSSÃO}

\section{CARACTERÍSTICAS FISIONÔMICAS}

A fitofisionomia desse ambiente florestal caracterizou-se por um grande número de árvores altas e finas, com mais de $50 \%$ dos indivíduos situando-se entre 14 e $25 \mathrm{~m}$ (Figura 2). Dos 670 espécimes amostrados, 467 apresentaram DAP $\leq 22,1$ $\mathrm{cm}$ (Figura 3), perfazendo $70 \%$ do total. Abarema mataybifolia (Sandw.) Barneby \& Grimes, Leonia glycycarpa Ruiz \& Pav., Swartzia reticulata Ducke e Aspidosperma oblongum A. DC., foram as únicas espécies a apresentarem valores superiores a $90 \mathrm{~cm}$ de DAP (dados não mostrados). 


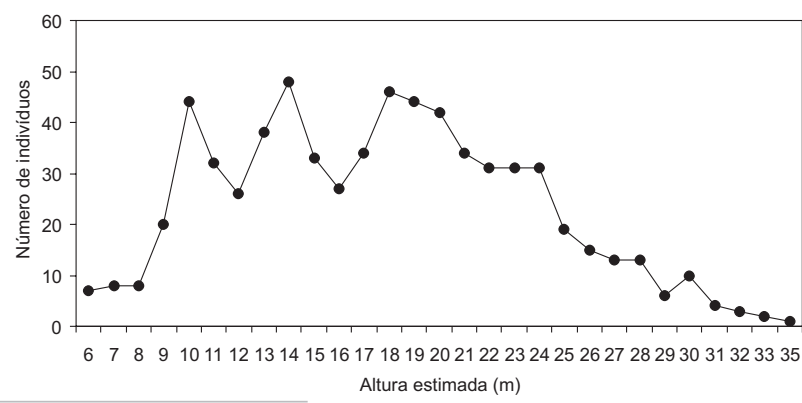

Figura 2 - Relação entre as alturas estimadas e o número de indivíduos amostrados no ambiente florestal estudado. Amazônia Central, Amazonas, Brasil.

A distribuição diamétrica dos indivíduos amostrados evidenciou uma curva decrescente (curva em forma de "J" invertido, Figura 3) que, segundo Tello (1995), Ferreira \& Prance (1998), Oliveira \& Mori (1999), Amaral et al. (2000), Lima Filho et al. (2001) e Oliveira \& Amaral (2004), é o comportamento típico de muitas florestas de terra firme da Amazônia Central. Portanto, o comportamento decrescente apresentado sugere que esse ambiente florestal, até o momento, não sofreu perturbação mais severa, o que é confirmado tanto pelo reduzido número de espécies pioneiras, como pela alta riqueza de espécies na amostragem.

O sub-bosque mostrou-se denso, com dominância de juvenis de Oenocarpus bacaba Mart., Astrocaryum sciophilum (Miq.) Pulle e Attalea sp., evidenciando a alta tolerância destas palmeiras à pouca luminosidade, conforme registrado por outros autores em florestas tropicais (Denslow, 1987; Matos \& Amaral, 1999; Oliveira \& Amaral, 2005). Observou-se ainda grande número de Protium apiculatum Swart, Inga bicoloriflora Ducke, Duguetia flagellaris Huber, Swartzia ulei Harms e Pouteria decorticans T.D. Penn. Ainda nesse estrato,

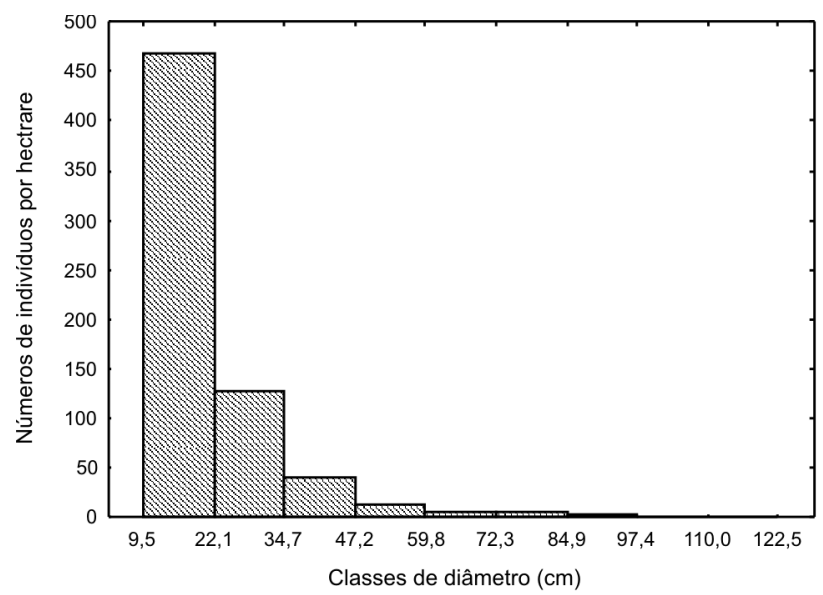

Figura 3 - Distribuição diamétrica (DAP $\geq 10 \mathrm{~cm}$ ) dos 670 indivíduos amostrados no ambiente florestal estudado. Amazônia Central, Amazonas, Brasil as espécies lenhosas mais frequentes, além das supracitadas, foram Eschweilera micrantha (Berg.) Miers, Chrysophyllum sanguinolentum (Pierre) Baehni e Licania davillifolia Benoist. Por sua vez, Ischnosiphon sp., Pariana cf campestris Aubl. e Heliconia acuminata Richard, foram as herbáceas mais expressivas do sub-bosque.

Nas clareiras fora dos transectos, observou-se a presença de cipós finos $(\mathrm{DAP} \leq 10 \mathrm{~cm})$ sendo melhor representados por Salacia insignis A.C. Sm. e Derris sp.; as epífitas pertencentes às aráceas, orquidáceas, bromeliáceas e pteridófitas foram as mais freqüentes. O estrato médio apresentou-se menos denso que o sub-bosque, sendo dominado por Protium apiculatum, Eschweilera micrantha, Licania davillifolia e Oenocarpus bacaba (dados não mostrados).

O dossel possui aspecto rugoso, com copas globosas, próximas uma das outras, resultando na pouca passagem de luz para os estratos inferiores. As espécies emergentes não ultrapassaram $35 \mathrm{~m}$ de altura (Figura 2), sendo Swartzia panacoco (Aubl.) R.S. Cowan, Dinizia excelsa Ducke, Cariniana decandra Ducke e Eschweilera micrantha (Berg.) Miers as mais frequentes (dados não mostrados).

\section{COMPOSIÇÃO FLORÍSTICA}

Foram anotados 670 indivíduos, distribuídos em 48 famílias, 133 gêneros e 245 espécies (Tabela 1). Considerando como espécies localmente raras aquelas que ocorrem na amostragem com apenas um indivíduo (Oliveira \& Amaral, 2004), pode-se inferir que nesse estudo, cerca de $49 \%$ (120) são raras na área amostrada, corroborando muitos trabalhos na região (Prance et al., 1976; Porto et al., 1976; Amaral, 1996; Oliveira \& Amaral, 2004) que registraram de 40 a $60 \%$ dessas espécies em seus levantamentos de 1 hectare.

Comparando a abundância das espécies com outros resultados no estado do Amazonas (Tello, 1995; Matos \& Amaral, 1999; Amaral et al., 2000; Lima Filho et al., 2001), notou-se que a área amostrada tem menor densidade por hectare. Apesar disso, a riqueza de taxa em termos de família, gênero e espécie, aproximou-se dos relatados naqueles estudos, superando inclusive alguns deles quanto à diversidade de espécies (Tabela 2).

Comparado aos levantamentos florísticos realizados na mesma estação experimental (Higuchi et al., 1998; Sakai et al., 2000), houve incremento médio de $12 \%$ sobre o número de espécies por hectare. Tal superioridade era esperada, uma vez que esses autores não consideraram as palmeiras e lianas lenhosas em suas amostragens.

A participação das lianas tanto na composição florística como na abundância, foi expressiva. Dos 670 espécimes levantados, as lianas contribuíram com $2,5 \%$. Nas famílias, das 48 anotadas no inventário, $18 \%$ possuem essa forma de vida, representando $5,3 \%$ das espécies identificadas. Fabaceae 
Tabela 1 - Relação das famílias, espécies, hábitos e seus interesses econômicos e/ou ecológico no ambiente florestal estudado. Amazônia Central, Amazonas, Brasil.

\begin{tabular}{|c|c|c|c|}
\hline Famílias & Espécies & Hábitos & Interesses** \\
\hline \multirow[t]{2}{*}{ Anacardiaceae } & Anacardium cf. microsepalum Loes. & árvore & ecológico \\
\hline & A. spruceanum Benth. ex Engl. ${ }^{*}$ & arvoreta & madeireiro/ecológico \\
\hline \multirow[t]{14}{*}{ Annonaceae } & Annona ambotay Aubl. ${ }^{*}$ & árvore & ecológico \\
\hline & Bocageopsis multiflora (Mart.) R.E. Fr. & $"$ & $"$ \\
\hline & Duguetia yeshidan Sandw. & $"$ & $"$ \\
\hline & Ephedranthus amazonicus R.E. Fr. & $"$ & madeireiro/ecológico \\
\hline & Fusaea Iongifolia (Aubl.) Saff. & $"$ & ecológico \\
\hline & Guatteria citriodora Ducke ${ }^{*}$ & $"$ & $"$ \\
\hline & G. discolor R.E. Fr. & $"$ & $"$ \\
\hline & G. olivacea R.E. Fr. & $"$ & $"$ \\
\hline & G. scytophylla Diels. & $"$ & $"$ \\
\hline & Guatteria sp. $1^{*}$ & $"$ & $"$ \\
\hline & Guatteria sp. $2^{*}$ & $"$ & $"$ \\
\hline & Guatteria sp. $3^{*}$ & $"$ & $"$ \\
\hline & Rollinia insignis R.E.Fr. & $"$ & $"$ \\
\hline & Unonopsis sp.* & $"$ & $"$ \\
\hline \multirow[t]{5}{*}{ Apocynaceae } & Aspidosperma oblongum A. DC. & $"$ & ecológico \\
\hline & Aspidosperma sp.. & $"$ & $"$ \\
\hline & Couma macrocarpa Barb. Rodr. & $"$ & comestível/ecológico \\
\hline & Himatanthus sp. & $"$ & ecológico \\
\hline & Lacmellea arborescens (Müll. Arg.) Markgr. & $"$ & " \\
\hline \multirow[t]{2}{*}{ Arecaceae } & Oenocarpus bacaba Mart. & palmeira & comestível/ecológico \\
\hline & 0. bataua Mart.* & " & $"$ \\
\hline \multirow[t]{2}{*}{ Bignoniaceae } & Jacaranda copaia (Aubl.) D. Don. & árvore & madeireiro/ecológico \\
\hline & Tabebuia incana Gentry* & $"$ & $"$ \\
\hline Bombacaceae & Scleronema micranthum Ducke & $"$ & $"$ \\
\hline \multirow[t]{7}{*}{ Burseraceae } & Protium apiculatum Swart & $"$ & $"$ \\
\hline & P. aracouchini (Aubl.) March. & $"$ & $"$ \\
\hline & P. decandrum (Aubl.) March. & " & " \\
\hline & P. hebetatum Daly & $"$ & $"$ \\
\hline & P. insigne (Triana \& Planch.) Engl.* & arvoreta & $"$ \\
\hline & Protium sp.* & árvore & $"$ \\
\hline & Tetragastris panamensis (Engl.) Kuntze & $"$ & $"$ \\
\hline \multirow[t]{13}{*}{ Caesalpiniaceae } & Bauhinia alata Ducke* & liana & medicinal/ecológico \\
\hline & Dimorphandra caudata Ducke & árvore & ecológico \\
\hline & Eperua bijuga Mart. ex Benth. & $"$ & $"$ \\
\hline & Macrolobium arenarium Ducke ${ }^{*}$ & árvore & $"$ \\
\hline & M. gracile Spruce ex Benth. & $"$ & $"$ \\
\hline & M. microcalyx Ducke & árvore & $"$ \\
\hline & Peltogyne excelsa Ducke & $"$ & madeireiro/ecológico \\
\hline & P. paniculata Benth & $"$ & $"$ \\
\hline & Sclerolobium guianense Benth. & $"$ & ecológico \\
\hline & Sclerolobium sp. $1^{\star}$ & $"$ & $"$ \\
\hline & Sclerolobium sp. $2^{*}$ & $"$ & $"$ \\
\hline & Sclerolobium sp. $3^{*}$ & $"$ & $"$ \\
\hline & Sclerolobium sp. $4^{*}$ & $"$ & $"$ \\
\hline Caryocaraceae & Caryocar glabrum (Aubl.) Pers. ${ }^{*}$ & $"$ & comestível/ecológico \\
\hline
\end{tabular}


Tabela 1 - Continuação

\begin{tabular}{|c|c|c|c|}
\hline Famílias & Espécies & Hábitos & Interesses** \\
\hline \multirow{4}{*}{ Cecropiaceae } & Cecropia sp.* & " & ecológico \\
\hline & Coussapoa latifolia Aubl.* & liana & $"$ \\
\hline & Pourouma bicolor Mart. ${ }^{*}$ & árvore & $"$ \\
\hline & P. guianensis Aubl.* & $"$ & $"$ \\
\hline Celastraceae & Goupia glabra Aubl. & $"$ & $"$ \\
\hline \multirow[t]{11}{*}{ Chrysobalanaceae } & Couepia Iongipendula Pilg. & $"$ & $"$ \\
\hline & Hirtella araguariensis Prance ${ }^{\star}$ & $"$ & $"$ \\
\hline & H. bicornis Mart. \& Succ. & $"$ & $"$ \\
\hline & Licania canescens Benoist. & $"$ & $"$ \\
\hline & L. caudata Prance & $"$ & $"$ \\
\hline & L. davillifolia Benoist. & $"$ & $"$ \\
\hline & L. densiflora Kleinhoonte. & $"$ & $"$ \\
\hline & L. heteromorpha Benth. & $"$ & $"$ \\
\hline & L. Iatifolia Benth. ex. Hook. & $"$ & $"$ \\
\hline & L. macrophylla Benth.* & $"$ & $"$ \\
\hline & Licania sp.* & $"$ & $"$ \\
\hline \multirow[t]{4}{*}{ Clusiaceae } & Clusia sp.* & $"$ & $"$ \\
\hline & Moronobea coccinea Aubl.* & $"$ & madeireiro/ecológico \\
\hline & Tovomita pyrifolia Planch. \& Triana* & $"$ & ecológico \\
\hline & T. schomburgkii Planch. \& Triana & $"$ & $"$ \\
\hline \multirow[t]{3}{*}{ Combretaceae } & Buchenavia parvifolia Ducke* & árvore & $"$ \\
\hline & Combretum laxum Aubl.* & liana & $"$ \\
\hline & Combretum sp.* & $"$ & $"$ \\
\hline Connaraceae & Connarus perrottetii (DC.) Planch. ${ }^{*}$ & árvore & $"$ \\
\hline \multirow[t]{2}{*}{ Dichapetalaceae } & Tapura guianensis Aubl.* & arvoreta & $"$ \\
\hline & T. amazonica Poepp. \& Endl. ${ }^{*}$ & " & $"$ \\
\hline Dilleniaceae & Doliocarpus brevipedicellatus Garcke & liana & medicinal/ecológico \\
\hline \multirow[t]{5}{*}{ Elaeocarpaceae } & Sloanea cf. guianensis (Aubl.) Benth. ${ }^{*}$ & árvore & ecológico \\
\hline & S. excelsa Ducke* & $"$ & $"$ \\
\hline & S. floribunda Spruce ex Benth.* & arvoreta & $"$ \\
\hline & S. pubescens Benth.* & árvore & $"$ \\
\hline & S. schomburgkii Spruce ex Benth. & $"$ & $"$ \\
\hline \multirow[t]{16}{*}{ Euphorbiaceae } & Conceveiba guianensis Aubl. & $"$ & $"$ \\
\hline & C. hostmannii Benth. & $"$ & $"$ \\
\hline & Croton lanjouwensis Jabl. & $"$ & $"$ \\
\hline & Hevea guianensis Aubl. & $"$ & $"$ \\
\hline & Mabea angularis G. den Hollander & $"$ & $"$ \\
\hline & M. cf. klugii Steyerm & $"$ & $"$ \\
\hline & Mabea sp. $1^{*}$ & $"$ & $"$ \\
\hline & Mabea sp. $2^{*}$ & $"$ & $"$ \\
\hline & M. speciosa Müll. Arg. & $"$ & $"$ \\
\hline & M. subsessilis Pax \& K. Hoffm. & $"$ & $"$ \\
\hline & Micrandra rossiana R.E. Schult. & $"$ & $"$ \\
\hline & Micrandra sp. & $"$ & $"$ \\
\hline & Micrandropsis scleroxylon W.A Rodrigues* & $"$ & madeireiro/ecológico \\
\hline & Nealchornea yapurensis Huber & $"$ & ecológico \\
\hline & Sandwithia guianensis Lanj. & $"$ & " \\
\hline & Senefeldera macrophylla Ducke & $"$ & $"$ \\
\hline
\end{tabular}


Tabela 1 - Continuação

\begin{tabular}{|c|c|c|c|}
\hline Famílias & Espécies & Hábitos & Interesses** \\
\hline \multirow[t]{21}{*}{ Fabaceae } & Andira parviflora Ducke & $"$ & madeireiro/ecológico \\
\hline & Bocoa viridiflora (Ducke) R.S. Cowan & $"$ & $"$ \\
\hline & Derris floribunda (Benth.) Ducke ${ }^{*}$ & liana & ecológico \\
\hline & Derris sp.* & $"$ & $"$ \\
\hline & Diplotropis purpurea (Rich.) Amshoff & árvore & madeireiro/ecológico \\
\hline & Dipteryx polyphylla Huber & $"$ & ecológico \\
\hline & Machaerium ferox Glaziou* & liana & $"$ \\
\hline & Machaerium sp.* & $"$ & $"$ \\
\hline & Ormosia sp.* & árvore & $"$ \\
\hline & Platymiscium duckei Huber* & $"$ & madeireiro/ecológico \\
\hline & Pterocarpus rohrii Vahl. & $"$ & $"$ \\
\hline & Pterocarpus sp.* & $"$ & $"$ \\
\hline & Swartzia apetala Raddi* & $"$ & ecológico \\
\hline & S. dolichopoda R.S. Cowan* & $"$ & $"$ \\
\hline & S. panacoco (Aubl.) R.S. Cowan & $"$ & $"$ \\
\hline & S. polyphylla DC. & $"$ & $"$ \\
\hline & S. recurva Poepp. & $"$ & $"$ \\
\hline & S. reticulata Ducke & $"$ & $"$ \\
\hline & S. schomburgkii Benth. & $"$ & $"$ \\
\hline & Swartzia sp.* & $"$ & $"$ \\
\hline & S. ulei Harms & $"$ & $"$ \\
\hline Hippocrateaceae & Tontelea cylindrocarpa (A.C. Sm.) A.C. Sm. & $"$ & $"$ \\
\hline \multirow[t]{4}{*}{ Humiriaceae } & Duckesia verrucosa (Ducke) Cuatrec. ${ }^{*}$ & $"$ & madeireiro/ecológico \\
\hline & Endopleura uchi (Huber) Cuatrec.* & $"$ & " \\
\hline & Sacoglottis guianensis Benth. & $"$ & ecológico \\
\hline & Vantanea guianensis (Aubl.) Ducke* & $"$ & $"$ \\
\hline \multirow[t]{12}{*}{ Lauraceae } & Aniba canelilla (H.B.K.) Mez. & $"$ & medicinal/ecológico \\
\hline & A. hostmanniana (Nees) Mez. ${ }^{*}$ & $"$ & madeireiro/ecológico \\
\hline & Aniba sp. $1^{*}$ & $"$ & $"$ \\
\hline & Aniba sp. $2^{*}$ & $"$ & $"$ \\
\hline & Dicypellium manausense W.A. Rodrigues & $"$ & $"$ \\
\hline & Licaria chrysophylla (Meissn.) Kosterm. & $"$ & $"$ \\
\hline & Licaria sp. & $"$ & $"$ \\
\hline & Mezilaurus itauba (Meissn.) Taub. ex Mez & $"$ & $"$ \\
\hline & Ocotea canaliculata (Rich.) Mez. ${ }^{*}$ & $"$ & $"$ \\
\hline & 0. myriantha (Meissn.) Mez* & $"$ & $"$ \\
\hline & Ocotea sp. $1^{\star}$ & $"$ & $"$ \\
\hline & Ocotea sp. $2^{*}$ & $"$ & $"$ \\
\hline \multirow[t]{8}{*}{ Lecythidaceae } & Cariniana decandra Ducke & $"$ & ecológico \\
\hline & Corythophora alta Knuth* & $"$ & madeireiro/ecológico \\
\hline & Eschweilera amazoniciformis S.A. Mori & $"$ & ecológico \\
\hline & E. atropetiolata S.A. Mori & $"$ & madeireiro/ecológico \\
\hline & E. bracteosa (Poepp. ex Berg) Miers & $"$ & ecológico \\
\hline & E. carinata S.A. Mori* & $"$ & $"$ \\
\hline & E. collina Eyma & $"$ & $"$ \\
\hline & E. coriacea (DC.) Mart. ex Berg. & $"$ & $"$ \\
\hline
\end{tabular}


Tabela 1 - Continuação

\begin{tabular}{|c|c|c|c|}
\hline \multirow[t]{11}{*}{ Famílias } & Espécies & Hábitos & Interesses** \\
\hline & E. decolorans Sandw. ${ }^{*}$ & " & $" 1$ \\
\hline & E. grandiflora (Aubl.) Sandw. ${ }^{*}$ & $"$ & $"$ \\
\hline & E. laevicarpa S.A. Mori* & arvoreta & $"$ \\
\hline & E. micrantha (Berg.) Miers & árvore & $"$ \\
\hline & E. pedicellata (Rich.) S.A. Mori* & árvore & $"$ \\
\hline & Eschweilera sp. 1 & $"$ & $"$ \\
\hline & Eschweilera sp. 2 & $"$ & $"$ \\
\hline & Eschweilera sp. $3^{*}$ & $"$ & $"$ \\
\hline & Gustavia hexapetala (Aubl.) Smith & $"$ & $"$ \\
\hline & Lecythis jarana A.C. Smith & $"$ & $"$ \\
\hline Loganiaceae & Strychnos jobertiana Baill.* & liana & $"$ \\
\hline \multirow[t]{5}{*}{ Meliaceae } & Guarea pubescens (Rich.) A. Juss. & árvore & $"$ \\
\hline & G. trunciflora C.DC. & $"$ & $"$ \\
\hline & Trichilia cf. micropetala Penn. & $"$ & $"$ \\
\hline & T. cipo (A .Juss.) C. DC.* & $"$ & $"$ \\
\hline & T. guianensis Klotz & $"$ & $"$ \\
\hline Memecylaceae & Mouriri huberi Cogn. & $"$ & $"$ \\
\hline Menispermaceae & Abuta candollei Triana \& Planch. & liana & $"$ \\
\hline \multirow[t]{9}{*}{ Mimosaceae } & Abarema mataybifolia (Sandw.) Barneby \& Grimes & $"$ & ecológico \\
\hline & Dinizia excelsa Ducke & $"$ & madeireiro/ecológico \\
\hline & Enterolobium sp.* & $"$ & $"$ \\
\hline & Inga alba (Sandw.) Willd. & $"$ & ecológico \\
\hline & I. capitata Desv. & $"$ & $"$ \\
\hline & I. obidensis Ducke* & $"$ & $"$ \\
\hline & I. paraensis Ducke & $"$ & $"$ \\
\hline & Zygia racemosa (Ducke) Barneby \& Grimes & $"$ & madeireiro/ecológico \\
\hline & Z. ramiflora (Benth.) Barneby \& Grimes & $"$ & ecológico \\
\hline \multirow[t]{14}{*}{ Moraceae } & Brosimum cf. guianense (Aubl.) Huber & $"$ & $"$ \\
\hline & B. rubescens Taub. & $"$ & madeireiro/ecológico \\
\hline & B. utile (H.B.K.) Pittier. & $"$ & ecológico \\
\hline & Clarisia racemosa Ruiz \& Pav. & $"$ & madeireiro/ecológico \\
\hline & Ficus sp. ${ }^{*}$ & arvoreta & " \\
\hline & Helianthostylis sprucei Baill. & árvore & ecológico \\
\hline & H. pedunculata Benoist. ${ }^{*}$ & $"$ & $"$ \\
\hline & Helicostylis sp.* & arvoreta & $"$ \\
\hline & Maquira calophylla (Planch. \& Endl.) Berg.* & árvore & $"$ \\
\hline & M. sclerophylla (Ducke) Berg. & $"$ & $"$ \\
\hline & Pseudolmedia laevis (Ruiz \& Pav.) Macbr.* & $"$ & madeireiro/ecológico \\
\hline & Sorocea muriculata Miq. & arvoreta & ecológico \\
\hline & Sorocea sp. & $"$ & $"$ \\
\hline & Trymatococcus amazonicus Poepp. \& Endl.. & árvore & $"$ \\
\hline \multirow[t]{6}{*}{ Myristicaceae } & Iryanthera dialyandra Ducke & $"$ & $"$ \\
\hline & Osteophloeum platyspermum (DC.) Warb.* & $"$ & madeireiro/ecológico \\
\hline & Virola caducifolia W.A Rodrigues* & $"$ & $"$ \\
\hline & V. calophylla (Spruce) Warb. & $"$ & $"$ \\
\hline & V. decorticans Ducke* & $"$ & $"$ \\
\hline & V. elongata (Benth.) Warb. & $"$ & $"$ \\
\hline
\end{tabular}




\begin{tabular}{|c|c|c|c|}
\hline Famílias & Espécies & Hábitos & Interesses** \\
\hline Myrsinaceae & Cybianthus pseudoicacoreus (Miq.) Agostini* & " & ecológico \\
\hline \multirow[t]{7}{*}{ Myrtaceae } & Calycolpus sp.* & $"$ & $"$ \\
\hline & Eugenia egensis DC.* & arvoreta & $"$ \\
\hline & E. patrisii Vahl* & árvore & $"$ \\
\hline & E. protenta Mc Vaugh & $"$ & $"$ \\
\hline & Eugenia sp.* & $"$ & $"$ \\
\hline & Myrcia cf. grandis McVaugh* & $"$ & $"$ \\
\hline & M. servata Mc Vaugh* & $"$ & $"$ \\
\hline \multirow[t]{2}{*}{ Nyctaginaceae } & Neea floribunda Poepp. \& Endl. & arvoreta & $"$ \\
\hline & N. oppositifolia Ruiz \& Pav. & árvore & $"$ \\
\hline \multirow[t]{4}{*}{ Olacaceae } & Aptandra tubicina (Poepp.) Benth. ex Miers* & arvoreta & $"$ \\
\hline & Heisteria barbata Cuatrc. ${ }^{*}$ & árvore & $"$ \\
\hline & H. scandens Ducke* & liana & $"$ \\
\hline & Minquartia guianensis Aubl. & $"$ & madeireiro/ecológico \\
\hline Polygalaceae & Moutabea guianensis Aubl.* & liana & ecológico \\
\hline \multirow[t]{3}{*}{ Quiinaceae } & Lacunaria crenata (Tul.) A.C. Sm. ${ }^{*}$ & árvore & $"$ \\
\hline & Quiina amazonica A.C. Sm.* & $"$ & $"$ \\
\hline & Touroulia amazonica Pires \& A.S. Foster ${ }^{*}$ & $"$ & $"$ \\
\hline Rosaceae & Prunus myrtifolia (L.) Urb. & árvore & $"$ \\
\hline \multirow[t]{3}{*}{ Rubiaceae } & Duroia macrophylla Huber & $"$ & $"$ \\
\hline & Ferdinandusa cf. uapuensis Spruce ex Schum* & $"$ & $"$ \\
\hline & F. elliptica Pohl.* & $"$ & $"$ \\
\hline \multirow[t]{3}{*}{ Sapindaceae } & Matayba arborescens (Aubl.) Radlk.. & $"$ & $"$ \\
\hline & Porocystis toulicioides Radlk. ${ }^{*}$ & $"$ & $"$ \\
\hline & Toulicia guianensis Aubl.* & $"$ & $"$ \\
\hline \multirow[t]{18}{*}{ Sapotaceae } & Chrysophyllum amazonicum Penn. & $"$ & $"$ \\
\hline & C. pomiferum (Eyma) Penn & $"$ & $"$ \\
\hline & C. sanguinolentum (Pierre) Baehni & $"$ & $"$ \\
\hline & C. ucuquirana-branca (Aubrév. \& Pellegr.) Penn. & $"$ & $"$ \\
\hline & Ecclinusa guianensis Eyma & $"$ & $"$ \\
\hline & Manilkara huberi (Ducke) A. Chev. & $"$ & madeireiro/ecológico \\
\hline & Micropholis guyanensis (DC.) Pierre & $"$ & ecológico \\
\hline & M. mensalis (Baehni) Aubrév. & $"$ & $"$ \\
\hline & M. venulosa (Mart. \& Eichler) Pierre* & $"$ & $"$ \\
\hline & Pouteria anomala (Pires) Penn. & $"$ & $"$ \\
\hline & P. glomerata (Miq.) Baehni* & arvoreta & $"$ \\
\hline & P. gongrijpii Eyma & árvore & $"$ \\
\hline & P. guianensis Aubl. & $"$ & $"$ \\
\hline & P. platyphylla (A.C. Sm.) Baehni & $"$ & $"$ \\
\hline & Pouteria sp. $1^{*}$ & $"$ & $"$ \\
\hline & Pouteria sp. $2^{*}$ & $"$ & $"$ \\
\hline & P. virescens Baehni ${ }^{\star}$ & $"$ & $"$ \\
\hline & Sarcaulus brasiliensis (DC.) Eyma.* & arvoreta & $"$ \\
\hline \multirow[t]{3}{*}{ Simaroubaceae } & Simaba cedron Planch.* & $"$ & $"$ \\
\hline & S. polyphylla (Cavalcante) W. Thomas* & árvore & $"$ \\
\hline & Siparuna decipiens (Tul.) DC. & $"$ & $"$ \\
\hline Sterculiaceae & Sterculia pruriens (Aubl.) Schum. & $"$ & $"$ \\
\hline
\end{tabular}




\begin{tabular}{|c|c|c|c|}
\hline Famílias & Espécies & Hábitos & Interesses** \\
\hline & Theobroma sylvestre Mart. & $"$ & $"$ \\
\hline Tiliaceae & Lueheopsis rosea (Ducke) Burret ${ }^{\star}$ & $"$ & $"$ \\
\hline Ulmaceae & Trema micrantha (L.) Blume* & $"$ & $"$ \\
\hline Verbenaceae & Vitex triflora Vahl. ${ }^{*}$ & arvoreta & $"$ \\
\hline \multirow[t]{5}{*}{ Violaceae } & Anphirrhox Iongifolia Spreng* & $"$ & $"$ \\
\hline & Leonia glycycarpa Ruiz \& Pav. & arvoreta & $"$ \\
\hline & Rinorea guianensis Aubl. & " & $"$ \\
\hline & R. paniculata (Mart.) Kuntze & $"$ & $"$ \\
\hline & R. racemosa (Mart.) Kuntze & arvoreta & $"$ \\
\hline Vochysiaceae & Erisma bicolor Ducke* & árvore & " \\
\hline
\end{tabular}

"Espécies com apenas um indivíduo na área amostrada. ${ }^{*}$ Baseado em Matos \& Amaral (1999) e Oliveira \& Amaral (2004).

e Combretaceae foram as mais representativas com 4 e 3 espécies, respectivamente (Tabela 1).

Estudos recentes indicam que, nas últimas décadas do século XX, a dominância de lianas em relação às árvores tem aumentado de 1,7 a 4,6\% ao ano em florestas da Amazônia (Phillips et al., 2002). De acordo com esses estudos, tal comportamento decorre, possivelmente, das mudanças climáticas no planeta, como o aumento da concentração de $\mathrm{CO}_{2}$ nas florestas tropicais. Maiores detalhes sobre essas mudanças e suas implicações sobre a composição e dinâmica das florestas tropicais podem ser encontrados nos trabalho de Condon et al. (1992), Laurance et al. (2001), Phillips et al. (2002) e Schnitzer \& Bongers (2002).

Diante dos resultados obtidos nesse estudo, pode-se inferir que, caso esse ambiente florestal sofra grandes perturbaçōes ou qualquer interferência antrópica desordenada, as lianas poderão dominar a composição florestal atual, uma vez que essa forma de vida é bastante agressiva, e de rápida colonização de áreas perturbadas (Laurance et al., 2001).

$\mathrm{Na}$ área, a participação das palmeiras foi inexpressiva na composição florística com DAP $\geq 10 \mathrm{~cm}$, contribuindo com apenas duas espécies: Oenocarpus bacaba Mart., com quatro indivíduos e $O$. bataua, com apenas um exemplar. A ausência de outras palmeiras de grande porte, tipicamente de florestas antropizadas, sugere que até o momento, esse ambiente florestal não sofreu qualquer tipo de perturbação, ratificando o comportamento em forma de $\mathrm{j}$ invertido observado para os 670 indivíduos amostrados (Figura 3).
As dez famílias que apresentaram maior riqueza de espécies foram: Fabaceae (21 espécies - 8,6\%), Sapotaceae (18 espécies - 7,3\%), Lecythidaceae (17 espécies - 6,9\%), Euphorbiaceae (16 espécies - 6,5\%), Annonaceae e Moraceae (14 espécies $5,7 \%$ ), Caesalpiniaceae (13 espécies - 5,3\%), Lauraceae (doze espécies - 4,9\%), Chrysobalanaceae (onze espécies - 4,5\%) e Mimosaceae (nove espécies - 3,7\%), correspondendo, em conjunto, a $60 \%$ das espécies identificadas. Esses resultados confirmam os inventários florísticos realizados em florestas de terra firme na Amazônia Central (Tello, 1995; Matos \& Amaral 1999; Amaral et al., 2000; Lima Filho et al., 2001; Oliveira \& Amaral, 2004), onde essas famílias destacam-se entre as dez mais diversificadas.

Quanto aos gêneros, Eschweilera (14 spp), Swartzia (nove spp), Licania e Pouteria (oito spp), Guatteria (sete spp), Protium e Mabea (seis spp), foram os mais representativos desse táxon. Excetuando-se Guatteria e Mabea, os demais gêneros ratificam outros estudos desenvolvidos na região (Tello, 1995; Matos \& Amaral 1999; Amaral et al., 2000; Oliveira \& Amaral, 2004).

As maiores abundâncias foram centralizadas nas famílias Lecythidaceae (102), Sapotaceae (75), Euphorbiaceae e Fabaceae (52), Burseraceae (41), Chrysobalanaceae (38), Annonaceae e Moraceae (33), Mimosaceae (30) e Lauraceae (26).

Comparando as famílias com maior riqueza específica e densidade, Caesalpiniaceae, com 25 indivíduos foi suplantada em densidade por Burseraceae. Apesar disso, manteve-se a

Tabela 2 - Comparação da diversidade florística entre a área estudada e outras localidades na Amazônia Central, Amazonas, Brasil.

\begin{tabular}{llllll}
\hline Localidades & No $^{0}$ famílias & NNo gêneros $^{\text {No espécies }}$ & No indivíduos & Autores \\
\hline Reserva Ducke & 48 & 134 & 142 & 719 & Tello (1995) \\
Região do rio Urucu & 44 & 125 & 253 & 710 & Amaral (1996) \\
Região próxima à Manaus & 47 & 138 & 285 & 618 & Oliveira \& Mori (1999) \\
Região do rio Uatumã & 47 & 118 & 145 & 720 & Amaral et al. (2000) \\
Região do rio Urucu & 48 & 122 & 322 & 769 & Lima Filho et al. (2001) \\
Estação experimental ZF-2 & 48 & 133 & 245 & 670 & Este estudo \\
\hline
\end{tabular}


relação registrada por vários autores na Amazônia (Campbell et al., 1986; Prance, 1990; Rankin-de-Merona et al., 1992; Amaral, 1996) entre riqueza de espécies e densidade nas famílias.

\section{ASPECTOS FITOSSOCIOLÓGICOS}

\section{ÍNDICES DE VALOR DE IMPORTÂNCIA (IVI)}

Entre as espécies amostradas, Eschweilera micrantha (Berg.) Miers apresentou o maior valor de IVI e conseqüentemente, a maior importância sociológica. Esse destaque deve-se a sua maior densidade e área basal, em relação às demais espécies. Porém, quando analisados os parâmetros de Aspidosperma oblongum A. DC., 9a espécie em IVI, observa-se que o valor obtido para área basal é o que melhor explica a dominância sociológica desta espécie, uma vez que apresentou baixas densidade $(0,30)$ e freqüência $(0,37)$ relativas (Tabela 3$)$.

Um outro aspecto interessante quanto a Eschweilera micrantha (Berg.) Miers, refere-se à dispersão dos seus indivíduos na área amostrada. Esta espécie mostrou-se relativamente bem distribuída ao longo da área estudada, tornando-se assim, possivelmente menos susceptível ao esgotamento genético (Matos \& Amaral, 1999). As outras, dentro de suas funçōes, são igualmente importantes para o equilíbrio ecológico desse ambiente florestal.

\section{ÍNDICES DE VALOR DE IMPORTÂNCIA FAMILIAR (IVIF)}

As dez famílias botânicas com maiores IVIF foram Lecythidaceae, Sapotaceae, Fabaceae, Euphorbiaceae, Chrysobalanaceae, Mimosaceae, Moraceae, Annonaceae, Burseraceae e Caesalpiniaceae (Figura 4). De um total máximo acumulado de $300 \%$ de IVIF, essas famílias responderam por $203 \%$, confirmando as observações quanto a esse índice para as florestas ombrófilas de terra firme da Amazônia Central (Matos \& Amaral, 1999; Oliveira \& Amaral, 2004).

Observa-se ainda que nem sempre a família com maior diversidade de espécies é a de maior importância na comunidade (Figura 4). Fabaceae com a maior diversidade relativa, $8,60 \%$, é considerada a terceira Família em importância sociológica. Entretanto, Lecythidaceae, com 6,50\% da diversidade relativa manifestou maior importância ecológica em relação às 48 famílias botânicas registradas. Esses resultados mostram que além da riqueza específica, os parâmetros densidade e dominância relativas, principalmente este último, são determinantes quando se avaliam as espécies florestais da região.

Tabela 3 - Distribuição em ordem decrescente das 20 espécies que mais se destacaram em Índice de Valor de Importância (IVI) no ambiente florestal estudado Amazônia Central, Amazonas, Brasil, onde ni = número de indivíduos por espécie; npo = número de parcelas que ocorreram as espécies; $\mathrm{DR}, \mathrm{FR}$ e DoR = valores em porcentagem.

\begin{tabular}{|c|c|c|c|c|c|c|c|c|c|c|}
\hline Espécies & $\mathrm{ni}$ & nро & G & $\mathrm{DA}$ & $\mathrm{DR}$ & FA & $\mathrm{FR}$ & DoA & DoR & IVI \\
\hline Eschweilera micrantha & 36 & 17 & 1,36 & 36 & 5,37 & 85 & 3,14 & 1,36 & 4,02 & 12,54 \\
\hline Pouteria gongrijpii & 19 & 10 & 1,01 & 19 & 2,84 & 50 & 1,85 & 1,01 & 2,99 & 7,67 \\
\hline Chrysophyllum sanguinolentum & 17 & 10 & 0,85 & 17 & 2,54 & 50 & 1,85 & 0,85 & 2,52 & 6,90 \\
\hline Protium apiculatum & 20 & 11 & 0,53 & 20 & 2,99 & 55 & 2,03 & 0,53 & 1,57 & 6,59 \\
\hline Eschweilera atropetiolata & 11 & 8 & 1,14 & 11 & 1,64 & 40 & 1,48 & 1,14 & 3,36 & 6,48 \\
\hline Swartzia reticulata & 9 & 7 & 0,96 & 9 & 1,34 & 35 & 1,29 & 0,96 & 2,83 & 5,47 \\
\hline Licania davillifolia & 11 & 5 & 0,97 & 11 & 1,64 & 25 & 0,92 & 0,97 & 2,87 & 5,44 \\
\hline Eschweilera coriacea & 14 & 8 & 0,50 & 14 & 2,09 & 40 & 1,48 & 0,50 & 1,49 & 5,06 \\
\hline Aspidosperma oblongum & 2 & 2 & 1,48 & 2 & 0,30 & 10 & 0,37 & 1,48 & 4,36 & 5,03 \\
\hline Dinizia excelsa & 7 & 6 & 0,87 & 7 & 1,04 & 30 & 1,11 & 0,87 & 2,57 & 4,73 \\
\hline Swartzia panacoco & 7 & 7 & 0,75 & 7 & 1,04 & 35 & 1,29 & 0,75 & 2,22 & 4,56 \\
\hline Licania latifolia & 10 & 9 & 0,41 & 10 & 1,49 & 45 & 1,66 & 0,41 & 1,22 & 4,37 \\
\hline Eschweilera amazoniciformis & 12 & 6 & 0,40 & 12 & 1,79 & 30 & 1,11 & 0,40 & 1,17 & 4,07 \\
\hline Protium aracouchini & 8 & 6 & 0,50 & 8 & 1,19 & 30 & 1,11 & 0,50 & 1,49 & 3,79 \\
\hline Scleronema micranthum & 8 & 5 & 0,52 & 8 & 1,19 & 25 & 0,92 & 0,52 & 1,52 & 3,64 \\
\hline Cariniana decandra & 3 & 3 & 0,83 & 3 & 0,45 & 15 & 0,55 & 0,83 & 2,46 & 3,46 \\
\hline Hevea guianensis & 6 & 5 & 0,49 & 6 & 0,90 & 25 & 0,92 & 0,49 & 1,46 & 3,28 \\
\hline Ecclinusa guianensis & 7 & 6 & 0,30 & 7 & 1,04 & 30 & 1,11 & 0,30 & 0,90 & 3,05 \\
\hline Leonia glycycarpa & 2 & 2 & 0,80 & 2 & 0,30 & 10 & 0,37 & 0,80 & 2,37 & 3,04 \\
\hline Senefeldera macrophylla & 10 & 4 & 0,25 & 10 & 1,49 & 20 & 0,74 & 0,25 & 0,74 & 2,97 \\
\hline Sub-totais & 219 & 137 & 14,92 & 219 & 32,67 & 685 & 25,30 & 14,92 & 44,13 & 102,14 \\
\hline Demais espécies & 451 & 404 & 18,93 & 451 & 67,33 & 2705 & 74,70 & 18,93 & 55,87 & 197,87 \\
\hline Totais & 670 & 541 & 33,85 & 670 & 100 & 2020 & 100 & 33,85 & 100 & 300 \\
\hline
\end{tabular}




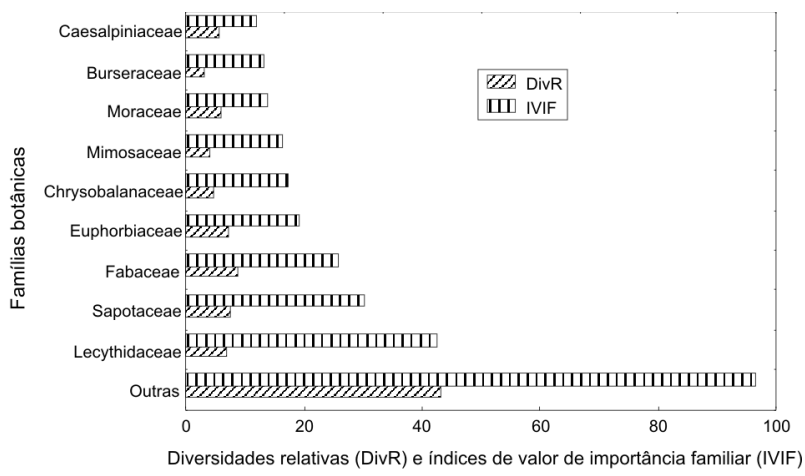

Figura 4 - Diversidades relativas (DivR) e Índices de Valor de Importância Familiar (IVIF) das dez famílias botânicas mais importantes do ambiente florestal estudado. Amazônia Central, Amazonas, Brasil.

\section{ÍNDICES ECOLÓGICOS}

A curva espécie-área indicou que a comunidade vegetal é muito diversa em espécies e insuficientemente amostrada com apenas um hectare (Figura 5), uma vez que esta não se estabilizou, ratificando muitos estudos realizados na Amazônia Central (Porto et al., 1976; Tello, 1995; Amaral, 1996; Amaral et al., 2000).

Entre as parcelas avaliadas, os índices de diversidade de Shannon-Wiener $\left(\mathrm{H}^{\prime}\right)$ variaram de 2,59 a 3,52 (Tabela 4). O índice médio de 3,19 de H' é considerado alto quando comparado com os valores de florestas temperadas, que normalmente variam de 2,0 a 3,0 (Knight, 1975). Por outro lado, para as espécies tropicais, principalmente da Amazônia Central, esse valor é baixo, uma vez que o menor índice já calculado para essa região foi de 3,59 (Porto et al., 1976). Para a área total, o H' foi de 5,10 (Tabela 4), indicando que esse ambiente apresenta grande diversidade florística, pois suplantou os limites máximos de 4,5 a 5,0 para as comunidades naturais (Margalef, 1972). Esse alto índice confirma a diversidade estimada a partir da curva espécie-área (Figura 5).

Quando se compara aos índices de outras florestas da região, com $\log _{\mathrm{e}} S$ e DAP mínimo de $10 \mathrm{~cm}$ (Porto et al., 1976; Tello, 1995, Higuchi et al., 1998; Oliveira \& Amaral, 2004), pode-se inferir que o índice observado nesse estudo é um dos maiores já documentados para a Amazônia Central, sendo superado apenas pela vegetação do Rio Urucu, na qual se observou valor de 5,28 (Amaral, 1996).

Pelo fato do H' ter sido reportado em poucos trabalhos com DAP de inclusão mínimo de $10 \mathrm{~cm}$, calculou-se o $\alpha$ de Fisher para fins de comparação com outros ambientes florestais de terra firme da Amazônia Central.

Os valores variaram de 53,01 ( $\mathrm{N}=719$ e $\mathrm{S}=142)$ a 208,3 $(\mathrm{N}=769$ e $\mathrm{S}=322)$. Observou-se que a alfa-diversidade obtida nesse estudo é inferior a de outras regiōes próximas aos Municípios de Manaus (Oliveira \& Mori 1999) e Coari (Lima Filho et al., 2001); porém, superior aos valores registrados para as outras comunidades vegetais. Excetuando o $\alpha$ de Fisher de 53,01 para a vegetação da Reserva Ducke, as demais comunidades sobre relevos mais ondulados (declives), apresentaram grande número de espécies em relação ao de indivíduos amostrados, resultando em algumas das maiores alfas-diversidades já registradas para a Amazônia (ter Steege, 2000).

Em relação aos índices de equitabilidade (uniformidade), houve maior uniformidade por parcela em relação à área total amostrada, sugerindo que a proporção indivíduo/espécie tende a se distribuir melhor em amostragens menores (Tabela 3).

O grau de 0,92 de equitabilidade estimado para a área amostrada (Tabela 4), indica que a distribuição das espécies dentro do povoamento é bastante uniforme, contradizendo o comportamento observado para a mesma área experimental, onde se registrou índice de 0,60, ou seja, distribuição apenas moderadamente uniforme (Higuchi et al., 1998).

As similaridades florísticas entre parcelas de 0,2 ha variaram de 11,4 a $31,7 \%$ para o coeficiente quantitativo de Porcentagem, sendo as parcelas 15 e 20 as mais similares entre si (Figura 6 a). Couepia longipendula Pilg., Doliocarpus brevipedicellatus Garcke, Eschweilera atropetiolata S.A. Mori, E. coriacea (DC.) Mart. ex. Berg., Guatteria scytophylla Diels, Hevea guianensis Aubl, Licania latifolia Benth. ex. Hook., Neea floribunda Poepp. \& Endl. e Pouteria gongrijpii Eyma, foram as espécies comuns às duas parcelas.

Para o coeficiente qualitativo de Sorensen, a variação foi de 13,0 a $34,4 \%$, com maior similaridade florística entre as parcelas 6 e 7 , terceiro maior pareamento para o coeficiente de Porcentagem (Figura $6 \mathrm{c}$ ). As espécies comuns entre as parcelas mais similares foram Bocageopsis multiflora (Mart.) R.E. Fr., Conceveiba guianensis Aubl., Dicypellium manauense W.A.

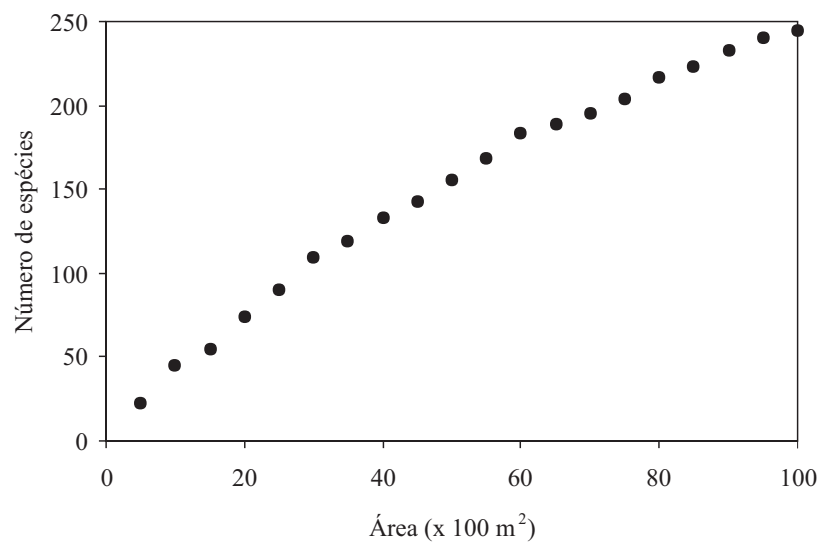

Figura 5 - Curva cumulativa do número de espécies por unidade amostral com DAP $\geq$ a $10 \mathrm{~cm}$ no ambiente florestal estudado. Amazônia Central, Amazonas, Brasil 
Tabela 4 - Índices de diversidade e equitabilidade de Shannon por parcela $\left(500 \mathrm{~m}^{2}\right)$ e área total $\left(10000 \mathrm{~m}^{2}\right)$ no ambiente florestal estudado. Amazônia Central, Amazonas, Brasil.

\begin{tabular}{lll}
\hline \multirow{2}{*}{ Parcelas } & \multicolumn{2}{c}{ Shannon-Wiener } \\
\cline { 2 - 3 } & Índices $\left(\mathrm{H}^{\prime}\right)$ & Equitabilidades $\left(\mathrm{E}^{\prime}\right)$ \\
\hline 1 & 2,93 & 0,95 \\
2 & 3,19 & 0,97 \\
3 & 2,59 & 0,91 \\
4 & 3,23 & 0,96 \\
5 & 3,16 & 0,98 \\
6 & 3,43 & 0,97 \\
7 & 3,38 & 0,99 \\
8 & 3,37 & 0,99 \\
9 & 3,05 & 0,97 \\
10 & 3,20 & 0,97 \\
11 & 3,20 & 0,98 \\
12 & 3,39 & 0,98 \\
13 & 2,97 & 0,98 \\
14 & 2,97 & 0,98 \\
15 & 3,29 & 0,98 \\
16 & 3,52 & 0,99 \\
17 & 2,90 & 0,99 \\
18 & 3,23 & 0,96 \\
19 & 3,39 & 0,99 \\
20 & 3,49 & 0,97 \\
Médias & 3,19 & 0,97 \\
Área total & 5,10 & 0,92 \\
\hline & & \\
\hline
\end{tabular}

Rodrigues, Dinizia excelsa Ducke, Eschweilera amazoniciformis S.A. Mori, E. micrantha (Berg.) Miers, Mabea speciosa Müll. Arg. e Micropholis guyanensis (DC.) Pierre.

Os agrupamentos formados a partir dos dois índices de similaridade seguiram um padrão praticamente idêntico, separando-se sempre em dois grupos bem definidos que correspondem aos dois transectos avaliados (Figura 6 a-d). Excetuando-se a parcela 9, as demais se encontram próximas entre si, com uma distância máxima de 250 m (Figura 6 a).

Segundo Campbell (1994) e Ferreira \& Prance (1998), em florestas de terra firme, as similaridades entre parcelas próximas são baixas, variando entre 10 a $36 \%$. Porém, nesse estudo constatou-se que quanto menor a distância geográfica entre parcelas de 0,2 hectare, maior será a similaridade florística observada (Figura 6 b-d). Esta constatação foi confirmada

Tabela 5 - Correlações estandardizadas de Mantel entre as similaridades florísticas e as distâncias geográficas na presença e ausência das espécies "localmente raras" no ambiente florestal estudado. Amazônia Central, Amazonas, Brasil.

\begin{tabular}{ll}
\hline Comunidade de plantas por hectare & Distâncias entre parcelas $(\mathrm{m})$ \\
\hline Com todas as espécies identificadas & $0,338 * * *$ \\
Sem as espécies "localmente raras" & $0,370 * * *$ \\
\hline
\end{tabular}
$* \star *=P<0,001$.
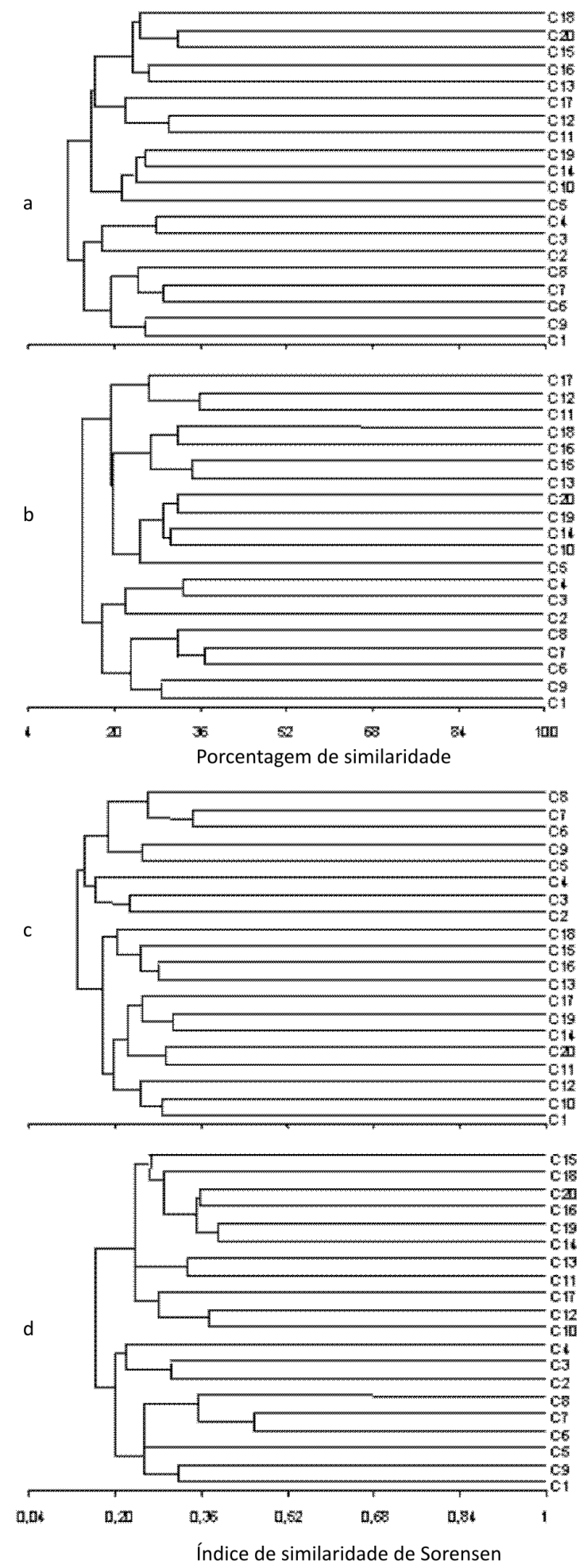

Figura 6 - Análises de agrupamento pelos índices de Porcentagem (a, b) e Sorensen (c, d) no ambiente florestal estudado. Amazônia Central, Amazonas, Brasil, onde a e $c=$ todas as espécies; b e $d=$ excluindo as espécies localmente raras. 
através do teste de Mantel, independente dos índices de similaridade aplicados, tanto na presença como na ausência das espécies "localmente raras" (Tabela 5).

A baixa similaridade registrada pode estar ainda relacionada ao alto percentual de espécies "localmente raras" presentes na amostragem (49\%). Com a retirada destas espécies da análise, observou-se que a dissimilaridade tende a diminuir entre as parcelas avaliadas, com as similaridades máximas aumentando em 5 e $12 \%$, para os índices de Porcentagem e Sorensen, respectivamente (Figura 6 b, d).

Esse comportamento sugere que a alta dissimilaridade florística entre comunidades vegetais de terra firme da Amazônia Central é independente do tamanho da área amostral, porém, estatisticamente dependente da distância entre locais de amostragem e do número de espécies localmente raras presentes no inventário florístico.

\section{BIBLIOGRAFIA CITADA}

Amaral, I.L. 1996. Diversidade Floristica em Floresta de Terra Firme, na regiāo do rio Urucu - AM. Dissertação de Mestrado. Instituto Nacional de Pesquisas da Amazônia/Universidade Federal do Amazonas, Manaus. 160pp.

Amaral, I.L.; Matos F.D.A.; Lima J. 2000. Composição florística e estrutural de um hectare de floresta densa de terra firme no Rio Uatumã, Amazônia, Brasil. Acta Amazonica, 30:377-392.

Campbell, D.D.; Daly, D.C.; Prance, G.T.; Maciel, U.N. 1986. Quantitative ecological inventory of terra firme and varzea tropical forest on the rio Xingu, Brazilian, Amazon. Brittonia, 38:369-393.

Campbell, D.C. 1994. Scale and patterns of community structure in Amazonian forests. In: P.J. Edwars.; R.M. May.; N.R. Webb. (eds.). Large-scale Ecology and Conservation Biology. Blackwell Scientific Publications, Oxford. pp. 179-198.

Chauvel, A. 1982. Os latossolos Amarelos, Álicos, Argilosos, dentro dos Ecossistemas das Bacias Experimentais do INPA e da Região Vizinha. Acta Amazonica, 12(3):47-60.

Clinebell, R.R.; Phillips, O.L.; Gentry, A.H.; Stark, N.; Zuuring, H. 1995. Prediction of neotropical tree and liana species richness from soil and climatic data. Biodiversity and Conservation, 4:56-90.

Condon, M.A.; Sasek, T.W.; Strain, B.R. 1992. Allocation patterns in two tropical vines in response to increased atmospheric $\mathrm{CO}_{2}$. Functional Ecology, 6:680-685.

Denslow, J.S. 1987. Tropical rainforest gaps and tree species diversity. Annual Review of Ecology and Systematics, 18:431-451.

Ferreira, L.V.; Prance, G.T. 1998. Species richness and floristic composition in four hectares in the Jaú National Park in upland forests in Central Amazonia. Biodiversity and Conservation, 7:1349-1364.

Gentry, A.H. 1988. Changes in plant community diversity and floristic composition on environmental and geographical gradients. Annals of the Missouri Botanical Garden, 75:1-34.
Guillaumet, J.L. 1987. Some structural and floristic aspects of the forest. Experientia, 43:241-251.

Higuchi, N.; Santos, J.;Vieira, G.; Ribeiro, R.J.; Sakurai, S.; Ishizuca, M.; Sakai, T.; Tanaka, N.; Saito, S. 1998. Plant structural analysis of a pristine tropical moist forest in Cuieiras river basin region, ZF - 2, Manaus - AM, Brazil. In: N. Higuchi.; M.A.A. Campos.; P.T.B. Sampaio; J. Santos (eds.). Pesquisas florestais para a conservação e reabilitação de áreas degradadas da Amazônia. Instituto Nacional de Pesquisas da Amazônia, Manaus. pp 53-81.

Knight, D.H. 1975. A phytosociological analysis of species-rich tropical forest on Barro Colorado Island, Panama. Ecological Monographs, 45:259-28.

Krebs, C.J. 1989. Ecological methodology. Harper \& Row, New York. 654pp.

Laurance, W.E.; Pérez-Salicrup, D.R.; Delamônica, P.; Fearnside, P.M.; Agra, S.; Jerozolinski, A.; Pohl, L. \& Lovejoy, T.E. 2001. Rain forests fragmentation and the structure of Amazonian liana communities. Ecology, 82:105-116.

Lima Filho, D.A.; Matos, F.D.A.; Amaral, I.L.; Revilla, J.; Coêlho, L.S.; Ramos, J.F.; Santos, J.L. 2001. Inventário florístico de floresta ombrófila densa de terra firme, na região do Rio UrucuAmazonas, Brasil. Acta Amazonica, 31:565-579.

Magurran, A.E. 1988. Ecological diversity and its measurement. Cambridge University Press, New Jersey. 197pp.

Mantel, N. 1967. The detection of Disease Clustering and a Generalized Regression Approach. Cancer Research, 72:209220.

Margalef, R. 1972. Homage to Evelyn Hutchinson, or why there is an upper limit to diversity. Transp. Connect. Acad. Arts Sci., 44:214-235.

Matos, F.D.A.; Amaral, I.L. 1999. Análise ecológica de um hectare em floresta ombrófila densa de terra-firme, estrada da várzea, Amazonas, Brasil. Acta Amazonica, 29:365-379.

Mori, A.S.; Boom, B. 1983. Ecological importance of Myrtaceae in na eastern Brazilian wet forest. Biotropica, 15:68-70.

Müller-Dombois, D.; Ellemberg, H. 1974. Aims and methods for vegetation ecology. John Wiley \& Sons, New York. 547pp.

Oliveira, A.A.; Mori, S.A. 1999. A central Amazonian terra firme forest. I. High tree species richness on poor soils. Biodiversity and Conservation, 8:1219-1244.

Oliveira, A.A.; Daly, D.C. 1999. Geographic distribution of tree species occurring in the region of Manaus, Brazil: implications for regional diversity and conservation. Biodiversity and Conservation, 8:1245-1259.

Oliveira, A.A. 2000. Inventários quantitativos de árvores em florestas de terra firme: Revisão com enfoque na Amazônia brasileira. Acta Amazonica, 30:543-567.

Oliveira, A.N.; Amaral, I.L. 2004. Florística e fitossociologia de uma floresta de vertente na Amazônia Central, Amazonas, Brasil. Acta Amazonica, 34:21-34.

Oliveira, A.N.;Amaral, I.L. 2005. Aspectos florísticos, fitossociológicos e ecológicos de um sub-bosque de terra firme na Amazônia Central, Amazonas, Brasil. Acta Amazonica, 35:1-16. 
Phillips, O.L.; Hall, P.; Gentry, A.H.; Sawyer, S.A.; Vásquez, R. 1994. amics and species richness of tropical rain forests. Proceedings of the Naional Academy of Sciences of the USA, 91:2805-2809.

Phillips, O.L.; Martínez, R.V.; Arroyo, L.; Baker, T.R.; Killeen, T.; Lewis, S.L.; Malhi, Y.; Mendoza, A.M.; Neill, D.; Núñez, P.; Alexiades, M.; Cerón, C.; DiFiore, A.; Erwin, T.; Jardim, A.; Palacios, W.; Saldias, M.; Vinceti, B. 2002. Increasing dominance of lianas in Amazonian forests. Nature, 418:770-774.

Porto, M.L.; Longhi, H.M.; Citadini, V.; Ramos, R.F.; Mariath J.E.A. 1976. Levantamento fitossociológico em área de "mata-debaixio", na estação Experimental de Silvicultura Tropical - INPA - Manaus - Amazonas. Acta Amazonica, 6:301-318.

Prance G.T., Rodrigues W.A.; Silva M.F. 1976. Inventário florestal de um hectare de mata de terra firme, $\mathrm{km} 30$ da estrada ManausItacoatiara. Acta Amazonica, 6:9-35.

Prance, G.T. 1990. The floristic composition of the forests of Central Amazonian Brazil. In: A. Gentry (ed.). Four Neotropical Forests. Yale University Press, New Haven, Connecticut. pp. 112-140.

Radam. 1978. Programa de Integração Nacional. Levantamentos de Recursos Naturais. v.18 (Manaus) - Radam (projeto) DNPM, Ministério das Minas e Energia. Brasil. 626pp.

Rankin-de-Mérona, J., Prance, G.T.; Hutchings, R.W.; Silva, M.F.; Rodrigues W.A. 1992. Preliminary results of a large-scale tree inventory of upland rain forest in the Central Amazon. Acta Amazonica, 22:485-492.

Ranzani, G. 1980. Identificação e caracterização de alguns solos da Estação Experimental de Silvicultura Tropical do INPA. Acta Amazonica, 10:7-41.
Ribeiro, J.E.L.S, Hopkins, M.J.G.; Vicentini, A.; Sothers, C.A.; Costa, M.A.S.; Brito, J.M.; Souza, M.A.D.; Martins, L.H.P.; Lohmann, L.G.; Assunção, P.A.C.L.; Pereira, E.C.; Silva, C.F.; Mesquita, M.R.; Procópio, L.C. 1999. Flora da Reserva Ducke. Guia de Identificação dasPlantas Vasculares de uma Floresta de Terra-firmena Amazônia Central. Instituto Nacional de Pesquisas da Amazônia, Manaus. 793pp.

Schnitzer, S.A.; Bongers, F. 2002. The ecology of lianas and their role in forests. Trends in Ecology \& Evolution, 17:223-230.

ter Steege, H.; Sabatier, D.; Castellanos, H.; Andel, T.V.; Duivenvoorden, J.; Oliveira, A.A.; Renske, E.; Lilwah, R.; Maas, P.; Mori S. 2000. A regional perspective: Analysis of Amazonian floristic composition and diversity that includes a Guyana Shield. In: H. ter Steege (ed.). Plant Diversity in Guyana: Whit recommendations for a National Protected Areas Strategy. The Tropenbos Foundation, Wageningen. pp. 19-32.

Tello, J.C.R. 1995. Aspectos fitossociológicos das comunidades vegetais de uma toposseqüência da Reserva Florestal Ducke do INPA. Tese de Doutorado. Instituto Nacional de Pesquisas da Amazônia/ Universidade Federal do Amazonas, Manaus. 335pp.

Tuomisto, H.; Ruokolainen, K., Kalliola, R., Linna, A., Danjoy, W.; Rodriguez, Z. 1995. Dissecting Amazonian biodiversity. Science, 269:63-66.

Valencia, R.; Balslev, H.; Paz Y Mino, G.C. 1994. High tree alphadiversity in Amazonian Ecuador. Biodiversity and Conservation, 3:21-28.

Recebido em 21/02/2008

Aceito em 18/06/2008 
